\title{
Nonabelian Algebraic Topology
}

\author{
Ronald Brown*
}

October 29, 2018

UWB Math Preprint 04.15

\begin{abstract}
This is an extended account of a short presentation with this title given at the Minneapolis IMA Workshop on ' $n$-categories: foundations and applications', June 7-18, 2004, organised by John Baez and Peter May.
\end{abstract}

\section{Introduction}

This talk gave a sketch of a book with the title Nonabelian algebraic topology being written under support of a Leverhulme Emeritus Fellowship (2002-2004) by the speaker and Rafael Sivera (Valencia) [6]. The aim is to give in one place a full account of work by R. Brown and P.J. Higgins since the 1970s which defines and applies crossed complexes and cubical higher homotopy groupoids. This leads to a distinctive account of that part of algebraic topology which lies between homology theory and homotopy theory, and in which the fundamental group and its actions plays an essential role. The reason for an account at this Workshop on $n$-categories is that the higher homotopy groupoids defined are cubical forms of strict multiple categories.

Main applications are to higher dimensional nonabelian methods for local-to-global problems, as exemplified by van Kampen type theorems. The potential wider implications of the existence of such methods is one of the motivations of this programme.

The aim is to proceed through the steps

$$
(\text { geometry }) \longrightarrow\left(\begin{array}{c}
\text { underlying } \\
\text { processes }
\end{array}\right) \longrightarrow(\text { algebra }) \longrightarrow(\text { algorithms }) \longrightarrow\left(\begin{array}{c}
\text { computer } \\
\text { implementation }
\end{array}\right) \text {. }
$$

\footnotetext{
*I am grateful to the IMA for support at this Workshop, and to the Leverhulme Trust for general support.
} 
The ability to do specific calculations, if necessary using computers, is seen as a kind of test of the theory, and one which also leads to seeking of new results; such calculations seem at this stage of the subject to require strict algebraic models of homotopy types. We obtain some nonabelian calculations, and it is this methodology which we term nonabelian algebraic topology. It is fortunate that higher categorical structures, and in particular higher groupoid structures, do give nonabelian algebraic models of homotopy types which allow some explicit calculation. They have also led to new algebraic constructions, such as a nonabelian tensor product of groups, of Lie algebras, and of other algebraic structures, with relations to homology of these structures (see the references in [3]).

\section{Background}

Topologists of the early 20th century dreamed of a generalisation to higher dimensions of the nonabelian fundamental group, for applications to problems in geometry and analysis for which group theory had been successful. The dream seemed to be shattered by the discovery that Čech's apparently natural 1932 generalisation of the fundamental group, the higher homotopy groups $\pi_{n}(X, x)$, were abelian in dimensions $\geqslant 2$. So 'higher dimensional groups' seemed to be just abelian groups, and the dream seemed to be a mirage.

Despite this, the relative homotopy groups $\pi_{n}(X, A, x)$ were found to be in general nonabelian for $n=2$, and as a result J.H.C. Whitehead in the 1940s introduced the term 'crossed module' for the properties of the boundary map

$$
\partial: \pi_{2}(X, A, x) \rightarrow \pi_{1}(A, x)
$$

and the action of $\pi_{1}(A, x)$ on $\pi_{2}(X, A, x)$. In investigating 'adding relations to homotopy groups' he proved the subtle result (call it Theorem $\mathrm{W}$ ) that if $X$ is obtained from $A$ by adding 2-cells, then $\pi_{2}(X, A, x)$ is the free crossed $\pi_{1}(A, x)$-module on the characteristic maps of the 2-cells. The proof used transversality and knot theory.

A potentially new approach to homotopy theory derived from the expositions in Brown's 1968 book [2] and Higgins' 1971 book [11], which in effect suggested that most of 1-dimensional homotopy theory can be better expressed in terms of groupoids rather than groups. This led to a search for the uses of groupoids in higher homotopy theory, and in particular for higher homotopy groupoids. The basic intuitive concept was generalising from the usual partial compositions of homotopy classes of paths to partial compositions of homotopy classes (of some form) of cubes. One aim was a higher dimensional version of the van Kampen theorem for the fundamental group. A search for such constructs proved abortive for some years from 1966.

However in 1974 we observed that Theorem W gave a universal property for homotopy in dimension 2, which was suggestive. It also seemed that if the putative higher dimensional 
groupoid theory was to be seen as a success it should at least recover Theorem W. But Theorem $\mathrm{W}$ is about relative homotopy groups! It therefore seemed a good idea for us to look at the relative situation in dimension 2 , that is, to start from a based pair $X_{*}=(X, A, C)$, where $C$ is a set of base points, and define a homotopy double groupoid $\rho\left(X_{*}\right)$ using maps of squares. The simplest, and most symmetric, possibility seemed to be to consider $R_{2}\left(X_{*}\right)$ as the set of maps $f: I^{2} \rightarrow X$ which take the edges of $I^{2}$ to the subspace $A$ and take the vertices to the set $C$, and then to form $\rho_{2}\left(X_{*}\right)$ as a quotient by homotopy rel vertices of $I^{2}$ and through elements of $R_{2}$. Amazingly, this gave a double groupoid, whose 1-dimensional part was the fundamental groupoid $\pi_{1}(A, C)$ on the set $C$. The proof is not entirely trivial, and uses a filling technique.

Previous to this, work with Chris Spencer in 1971-2 had investigated the notion of double groupoid, shown a clear relation to crossed modules, and introduced the notion of connection on a double groupoid so as to define a notion of commutative cube in a double groupoid, generalising the notion of commutative square in a groupoid. Once the construction of $\rho_{2}\left(X_{*}\right)$ had been given, a proof of a 2-dimensional van Kampen theorem came fairly quickly. The paper on this was submitted in 1975, and appeared in 1978 [5] (after a third referee's report, the reports of the first two referees being withheld). The equivalence between double groupoids with connection and crossed modules established by Brown and Spencer [7], and so relating the double groupoid $\rho_{2}\left(X_{*}\right)$ to the second relative homotopy groups, enabled new calculations of some nonabelian second relative homotopy groups in terms of the associated crossed module, and so enabled calculation of some homotopy 2-types.

Thus there is considerable evidence that the nonabelian fundamental group is naturally generalised to various forms of crossed modules or double groupoids, and that these structures enable new understanding and calculations in dimension 2. The important point is that in dimension 2 we can easily define homotopical functors, that is, functors defined in terms of homotopy classes of maps, in a manner analogous to that of the fundamental group and groupoid, and then prove, but not so easily, theorems which enable us to calculate directly and exactly with these functors without using homological techniques.

As an example, we give the following problem. Given a morphism $\iota: P \rightarrow Q$ of groups, calculate the homotopy 2-type of the mapping cone $X$ of the induced map of classifying spaces $B \iota: B P \rightarrow B Q$. This 2-type can be described completely in terms of the crossed module $\partial: \iota_{*}(P) \rightarrow Q$ 'induced' from the identity crossed module $1: P \rightarrow P$ by the morphism $\iota[8]$.

There are some general results on calculating induced crossed modules, while for some calculations, we have had to use a computer. The following table, taken from [8], contains the results of computer calculations, using the package XMOD [1] in the symbolic computation system GAP [9]. The examples are for $Q=S_{4}$ and various subgroups $P$ of $Q$. The computer has of course full information on the morphisms $\partial: \iota_{*}(P) \rightarrow S_{4}$ in terms of generators of the groups in the table. The third column gives $\pi_{2}=\operatorname{Ker} \partial=\pi_{2}(X)$. The fourth column gives $\pi_{1}=$ Coker $\partial=\pi_{1}(X)$. The numbers 128.2322 and 96.67 refer to groups in the GAP4 table of 
groups.

\begin{tabular}{||c|c|c|c||}
\hline$P$ & $\iota_{*} P$ & $\pi_{2}$ & $\pi_{1}$ \\
\hline \hline$\langle(1,2)\rangle$ & $G L(2,3)$ & $C_{2}$ & 1 \\
$S_{3}$ & $G L(2,3)$ & $C_{2}$ & 1 \\
$\langle(1,2),(3,4)\rangle$ & $S_{4} C_{2}$ & $C_{2}$ & 1 \\
$D_{8}$ & $S_{4} C_{2}$ & $C_{2}$ & 1 \\
$C_{4}$ & 96.67 & $C_{4}$ & 1 \\
$C_{3}$ & $C_{3} S L(2,3)$ & $C_{6}$ & $C_{2}$ \\
$\langle(1,2)(3,4)\rangle$ & 128.2322 & $C_{4} C_{2}^{3}$ & $S_{3}$ \\
\hline
\end{tabular}

In fact the pairs of equal groups in the second column give pairs of isomorphic crossed modules, though the proof of this needs some calculation.

It is not claimed that these results are in themselves significant. But they do show that: (i) there are feasible algorithms; (ii) the homotopy groups, even with their structure as modules over the fundamental group, represent but a pale shadow of the actual homotopy type; (iii) this homotopy type can sometimes be represented by convenient nonabelian structures; (iv) we can use strict structures for explicit calculations in homotopy theory; (v) the colimit formulation allows for some complete calculations, not just up to extension; (vi) there is still the problem of determining the triviality or not of the first $k$-invariant in $H^{3}\left(\pi_{1}, \pi_{2}\right)$ for the last two examples.

It may be argued that the use of crossed modules has not yet been extended to the geometric and analytic problems which we have described above as motivating the need for a higher dimensional version of the fundamental group and groupoid. This argument shows that there is work to be done! Indeed, there are few books on algebraic topology other than [2] which mention even the fundamental groupoid on a set of base points.

Once the definitions and applications of the homotopy double groupoid of a based pair of spaces had been developed, it was easy to guess a formulation for a homotopy groupoid and GvKT in all dimensions, namely replace:

the based pair by a filtered space;

the crossed module functor by a crossed complex functor;

and the homotopy double groupoid by a corresponding higher homotopy groupoid.

However the proofs of the natural conjectures proved not easy, requiring developments in both the algebra of higher dimensional groupoids and in homotopy theory.

\section{Main results}

Major features of the work over the years with Philip J. Higgins and others can be summarised in the properties of the following diagram of categories and functors: 


\section{Diagram 2.1}

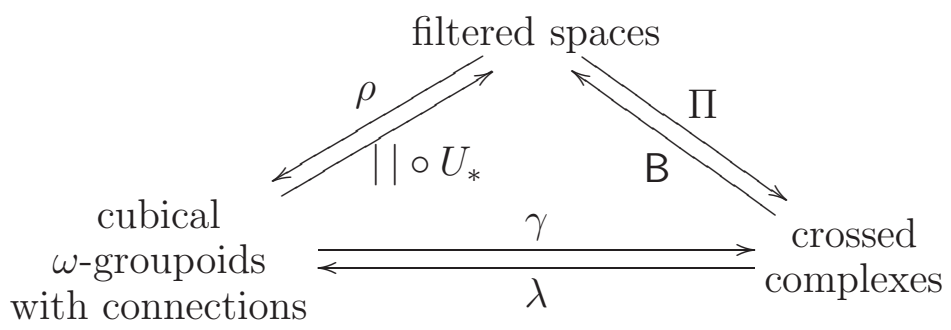

Let us first say something about the categories FTop of filtered spaces, and $\omega$-Gpds of cubical $\omega$-groupoids with connections. A filtered space consists of a (compactly generated) space $X$ and an increasing sequence of subspaces

$$
X_{*}: X_{0} \subseteq X_{1} \subseteq X_{2} \subseteq \cdots \subseteq X_{n} \subseteq \cdots \subseteq X
$$

With the obvious morphisms, this yields the category FTop.

One example of a filtered space is a manifold equipped with a Morse function. This example is studied using crossed complex techniques in [12, Chapter VI] (free crossed complexes are there called homotopy systems).

Another, and more standard, example of a filtered space is a $C W$-complex with its skeletal filtration. In particular, the $n$-cube $I^{n}$, the product of $n$-copies of the unit interval $I=[0,1]$, with its standard cell structure, becomes a filtered space $I_{*}^{n}$, and so for a filtered space $X_{*}$ we can define its filtered cubical singular complex $R^{\square}\left(X_{*}\right)$ which in dimension $n$ is simply $\operatorname{FTop}\left(I_{*}^{n}, X_{*}\right)$.

Thus $R^{\square}\left(X_{*}\right)$ is an example of a cubical set: it has for $n \geqslant 1$ and $i=1, \ldots, n$, face maps $\partial_{i}^{ \pm}$from dimension $n$ to dimension $(n-1)$, and in the reverse direction degeneracy maps $\varepsilon_{i}$, all satisfying a standard set of rules. We also need in the same direction as the $\varepsilon_{i}$ an additional degeneracy structure, called connections, $\Gamma_{i}^{ \pm}, i=1, \ldots, n$, which are defined by the monoid structures max, min on $I$. There are also in dimension $n$ and for $i=1, \ldots, n$ partially defined compositions $\circ_{i}$ given by the usual gluing $a \circ_{i} b$ of singular $n$-cubes $a, b$ such that $\partial_{i}^{+} a=\partial_{i}^{-} b$.

There is an equivalence relation $\equiv$ on $R^{\square}\left(X_{*}\right)_{n}$ given by homotopy through filtered maps rel vertices of $I^{n}$. This gives a quotient map

$$
p: R^{\square}\left(X_{*}\right) \rightarrow \rho^{\square}\left(X_{*}\right)=\left(R^{\square}\left(X_{*}\right) / \equiv\right) .
$$

It is easily seen that $\rho^{\square}\left(X_{*}\right)$ inherits the structure of cubical set with connections. A major theorem is that the compositions $\circ_{i}$ are also inherited, so that $\rho^{\square}\left(X_{*}\right)$ becomes a cubical $\omega$ groupoid with connections. The proof uses techniques of collapsing on subcomplexes of the $n$-cube. A development of these techniques to chains of partial boxes proves the surprising result, essential for the theory, that the quotient map $p$ of $(*)$ is a cubical Kan fibration. This 
can be seen as a rectification result, by which we mean a result using deformations to replace equations up to homotopy by strict equations.

An advantage of the functor $\rho$ and the category $\omega$-Gpds is that in this context we can prove a Generalised van Kampen Theorem (GvKT), that the functor $\rho$ preserves certain colimits. This yields precise calculations in a way not obtainable with exact or spectral sequences.

The proof is 'elementary' in the sense that it does not involve homology or simplicial approximation. However the proof is elaborate. It depends heavily on the algebraic equivalence of the categories $\omega$-Gpds and Crs of crossed complexes, by functors $\gamma, \lambda$ in the Main Diagram. This equivalence enables the notion of commutative cube in an $\omega$-groupoid, and the proof that any composition of commutative cubes is commutative. It is also shown that $\gamma \rho$ is naturally equivalent to a functor $\Pi$ defined in a classical manner using the fundamental groupoid and relative homotopy groups, as detailed below.

In detail we set $\Pi\left(X_{*}\right)_{1}=\pi_{1}\left(X_{1}, X_{0}\right)$, the fundamental groupoid of $X_{1}$ on the set of base points $X_{0}$, and for $n \geqslant 2$ we let $\Pi\left(X_{*}\right)_{n}$ be the family of relative homotopy groups $\pi_{n}\left(X_{n}, X_{n-1}, x\right), x \in X_{0}$. Setting $C_{0}=X_{0}$ and $C_{n}=\Pi\left(X_{*}\right)_{n}$ we find that $C$ obtains the structure of crossed complex,

\section{Diagram 2.2}

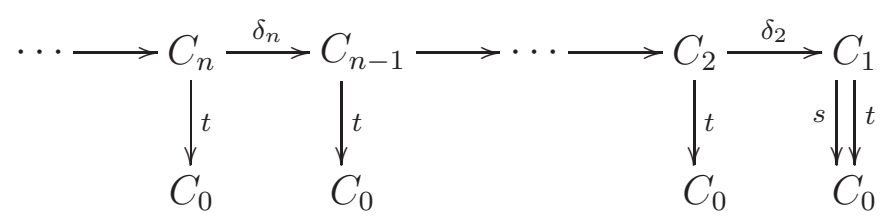

where the structure and axioms are those universally satisfied by this example $\Pi\left(X_{*}\right)$.

If $X_{*}$ is the skeletal filtration of a reduced $C W$-complex $X$, then $\Pi\left(X_{*}\right)$ should be regarded as a powerful replacement for the cellular chains of the universal cover of $X$, with operators from the fundamental group of $X$. However the use of crossed complexes rather than the cellular chains allows for better realisation properties. Also the functor $\Pi$ allows for many base points, so that groupoids are used in an essential way.

The GvKT for $\rho$ immediately transfers to a GvKT for this classical functor $\Pi$. We again emphasise that the proof of the GvKT for $\Pi$ is elaborate, and tightly knit, but elementary, in that it requires no background in homology, or simplicial approximation. It is a direct generalisation of the proof of the van Kampen Theorem for the fundamental groupoid. Indeed it was the intuitions that such a generalisation should exist that gave in 1966 the impetus to the present theory.

With this GvKT we deduce in the first instance:

- the usual vKT for the fundamental groupoid on a set of base points; 
- the Brouwer degree theorem $\left(S^{n}\right.$ is $(n-1)$-connected and $\left.\pi_{n} S^{n}=\mathbb{Z}\right)$;

- the relative Hurewicz theorem;

- Whitehead's theorem that $\pi_{2}\left(A \cup\left\{e_{\lambda}^{2}\right\}_{\lambda}, A, x\right)$ is a free crossed $\pi_{1}(A, x)$-module;

- a more general excision result on $\pi_{n}(A \cup B, A, x)$ as an induced module (crossed module if $n=2)$ when $(A, A \cap B)$ is $(n-1)$-connected;

- computations of $\pi_{n}(A \cup B, A \cap B, x)$ when $(A, A \cap B),(B, A \cap B)$ are $(n-1)$-connected.

Whitehead's theorem, and the last two results for $n=2$, are nonabelian results, and hence not obtainable easily, if at all, by homological methods.

The assumptions required of the reader are quite small, just some familiarity with $C W$ complexes. This contrasts with some expositions of basic homotopy theory, where the proof of say the relative Hurewicz theorem requires knowledge of singular homology theory. Of course it is surprising to get this last theorem without homology, but this is because it is seen as a statement on the morphism of relative homotopy groups

$$
\pi_{n}(X, A, x) \rightarrow \pi_{n}(X \cup C A, C A, x) \cong \pi_{n}(X \cup C A, x)
$$

and is obtained, like our proof of Whitehead's theorem, as a special case of an excision result. The reason for this success is that we use algebraic structures which model the geometry and underlying processes more closely than those in common use.

The cubical classifying space $B^{\square} C$ construction for a crossed complex $C$ is given by the geometric realisation of a cubical nerve $N^{\square} C$ defined by

$$
\left(N^{\square} C\right)_{n}=\operatorname{Crs}\left(\Pi\left(I_{*}^{n}\right), C\right) .
$$

The crossed complex $C$ has a filtration $C^{*}$ by successive truncations, and this gives rise to the filtered space $\mathrm{B}(C)=B^{\square} C^{*}$ for which there is a natural isomorphism $\Pi \mathrm{B}(C) \cong C$. The fundamental groupoid and homotopy groups of $B^{\square} C$ are just the fundamental groupoid and 'homotopy groups' of $C$. In particular, if $X_{*}$ is the skeletal filtration of a $C W$-complex $X$, and $C=\Pi\left(X_{*}\right)$, then $\pi_{1}(C) \cong \pi_{1}\left(X, X_{0}\right)$ and for $n \geqslant 2, \pi_{n}(C, x) \cong H_{n}\left(\widetilde{X}_{x}\right)$, the homology of the universal cover of $X$ based at $x$.

The category FTop has a monoidal closed structure with tensor product given by

$$
\left(X_{*} \otimes Y_{*}\right)_{n}=\bigcup_{p+q=n} X_{p} \times Y_{q} .
$$

It is also fairly easy to define a monoidal closed structure on the category $\omega$-Gpds since this category is founded in the structure on cubes. The equivalence of this category with that of 
crossed complexes, Crs, then yields a monoidal closed structure on the latter category, with exponential law

$$
\operatorname{Crs}(A \otimes B, C) \cong \operatorname{Crs}(A, \operatorname{CRS}(B, C))
$$

The elements of $\operatorname{CRS}(B, C)$ are: in dimension 0 just morphisms $B \rightarrow C$; in dimension 1 , are homotopies of morphisms; and in dimensions $\geqslant 2$ are forms of 'higher homotopies'. There is a complicated formula for the tensor product $A \otimes B$, as generated in dimension $n$ by elements $a \otimes b, a \in A_{p}, b \in B_{q}, p+q=n$, with a set of relations and boundary formulae related to the cellular decomposition of a product of cells $E^{p} \times E^{q}$, where $E^{p}$ has for $p>1$ just 3 cells of dimensions $0, p-1, p$ respectively.

Using the filtered 1-cube $I_{*}^{1}$, and the values on this of $\rho$ and $\Pi$, we have unit interval objects in each of the categories FTop, $\omega$-Gpds, Crs. This, with the tensor product, gives a cylinder object and so gives a homotopy theory on these categories.

Another difficult result is that the functor $\Pi$ preserves certain tensor products, for example of $C W$-filtrations. This result is needed to deduce the homotopy addition lemma for a simplex by induction, and also to prove the homotopy classification results.

A major result is that there is a natural bijection of homotopy classes

$$
\left[\Pi\left(X_{*}\right), C\right] \cong\left[X, B^{\square} C\right]
$$

for any crossed complex $C$ and $C W$-complex $X$, with its skeletal filtration. This is a special case of a weak equivalence

$$
B^{\square}\left(C R S\left(\Pi\left(X_{*}\right), C\right)\right) \rightarrow\left(B^{\square} C\right)^{X} .
$$

In fact the published version of this is for the simplicial classifying space $B^{\Delta} C$, and this has given also an equivariant version of these results.

In this theory the homotopy addition lemma (HAL) for a simplex falls out by a simple inductive calculation, since the usual representation of the $n$-simplex $\Delta^{n}$ as a cone on $\Delta^{n-1}$ is exactly modelled in the crossed complex case, where the cone is defined using the tensor product. The explicit formulae for the tensor product, and so for any cone construction, give an easy calculation of the HAL formula.

There is an acyclic model theory for crossed complexes similar to that for chain complexes. So we obtain an Eilenberg-Zilber type theorem for crossed complexes. This has been developed with explicit formulae by Tonks.

\section{Conclusion}

The book that is being written is divided into three parts, of which Part I is now available on the web [6]. Part I deals with the case of dimensions 1 and 2, and the proof and applications 
of the GvKT in this dimension. The reasons for this are that the step from dimension 1 to dimension 2 is a big one, and the reader needs to grasp the new ideas and techniques. Also a gentle introduction is needed to calculating with the (nonabelian) crossed modules.

Part II should be regarded as a kind of handbook on crossed complexes. The main properties of crossed complexes and of the functor $\Pi$ are stated. Applications and calculations are deduced. Thus crossed complexes are presented as a basic tool in algebraic topology.

The proofs of these basic properties of crossed complexes, such as the monoidal closed structure, and the GvKT, require however the use of the category of cubical w-groupoids with connection. The theory of these is developed in Part III.

Appendices give some basic theory needed, such as some category theory (adjoint functors, colimits, etc.).

A question asked at the Workshop was: Why concentrate on filtered spaces? The only answer I can give is that they provide a workable basis for a theory of higher homotopy groupoids. A more general theory, in some ways, is given by $n$-cubes of spaces, and the associated cat ${ }^{n}$ groups, but this does not have such an intuitive exposition as can be provided for the filtered case. Currently, there is in the absolute case no theory which works in dimensions $>2$, and even the known dimension 2 case does not yet provide a range of explicit calculations.

Peter May pointed out that, in contrast to stable homotopy theory, this work brings the fundamental group fully into the algebraic topology setting, and that such input is surely needed for applications in algebraic geometry. Note that Grothendieck's increasingly influential manuscript 'Pursuing Stacks' [10] was intended as an account of nonabelian homological algebra (private communication). But that subject should in principle need 'nonabelian algebraic topology' as a precursor.

The book makes no attempt to explain the work on cat $^{n}$-groups, which allows for calculations in homotopy theory which are more complex, and indeed more nonabelian, than those given in the planned book. That work thus continues the story of Nonabelian Algebraic Topology, and references to many authors and papers are given in [3].

The references which follow include some survey articles with wider references. We conclude with a diagram of historical context for the current theory ${ }^{1}$.

\section{References}

[1] M. Alp and C. D. Wensley, XMod, Crossed modules and Cat ${ }^{1}$-groups: a GAP4 package, (2004) (http://www.maths. bangor.ac.uk/chda/)

\footnotetext{
${ }^{1}$ I am grateful to Aaron Lauda for rendering this diagram in xypic.
} 
[2] R. Brown, Elements of Modern Topology, McGraw Hill, Maidenhead, 1968. second edition as Topology: a geometric account of general topology, homotopy types, and the fundamental groupoid, Ellis Horwood, Chichester (1988) 460 pp.

[3] R. Brown, 'Higher dimensional group theory', http://www.bangor.ac.uk/ mas010/hdaweb2.htm

[4] R. Brown, 'Crossed complexes and homotopy groupoids as non commutative tools for higher dimensional local-to-global problems', Proceedings of the Fields Institute Workshop on Categorical Structures for Descent and Galois Theory, Hopf Algebras and Semiabelian Categories, September 23-28, 2002, Contemp. Math. (2004). (to appear), UWB Math Preprint 02.26.pdf (30 pp.)

[5] R. Brown and P. J. Higgins, On the connection between the second relative homotopy groups of some related spaces, Proc.London Math. Soc., (3) 36 (1978) 193-212.

[6] R. Brown and R. Sivera, 'Nonabelian algebraic topology', (in preparation) Part I is downloadable from

(http://www. bangor.ac.uk/〜mas010/nonab-a-t.html)

[7] R. Brown and C. B. Spencer, Double groupoids and crossed modules, Cahiers Top. Géom. Diff., 17 (1976) 343-362.

[8] R. Brown and C. D. Wensley, 'Computation and homotopical applications of induced crossed modules', J. Symbolic Computation, 35 (2003) 59-72.

[9] The GAP Group, 2004, GAP - Groups, Algorithms, and Programming, version 4.4, Technical report, (http://www.gap-system.org)

[10] A. Grothendieck, 'Pursuing Stacks', 600p, 1983, distributed from Bangor. Now being edited by G. Maltsiniotis for the SMF.

[11] P. J. Higgins, 1971, Categories and Groupoids, Van Nostrand, New York. Reprint Series, Theory and Appl. Categories (to appear).

[12] V. Sharko, 1993, Functions on manifolds: algebraic and topological aspects, number 131 in Translations of Mathematical Monographs, American Mathematical Society.

Professor Emeritus R. Brown,

Department of Mathematics, University of Wales, Bangor, Dean St, Bangor, Gwynedd LL57 1UT, United Kingdom http://www.bangor.ac.uk/ mas010

email: r.brown@bangor.ac.uk 


\section{Some Context for Nonabelian Algebraic Topology}

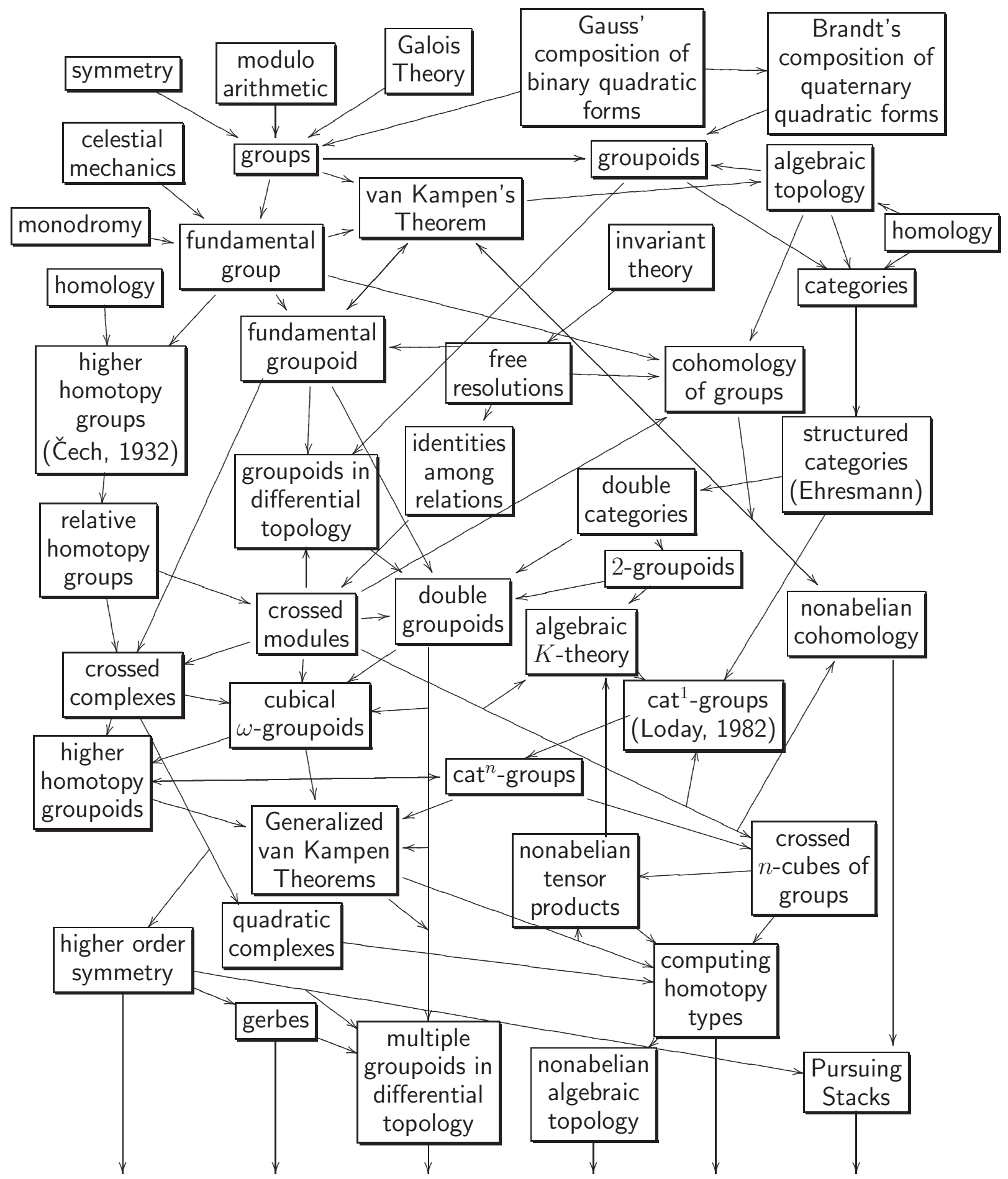

